Portland State University

PDXScholar

$1-1-2004$

\title{
Environmental Swap Energy and Role of Configurational Entropy in Transfer of Small Molecules from Water into Alkanes
}

\author{
Pavel Smejtek \\ Portland State University \\ Robert Campbell Word \\ Portland State University
}

Follow this and additional works at: https://pdxscholar.library.pdx.edu/phy_fac

Part of the Physics Commons

Let us know how access to this document benefits you.

\section{Citation Details}

Smejtek, P., \& Word, R. C. (2004). Environmental swap energy and role of configurational entropy in transfer of small molecules from water into alkanes. Journal Of Chemical Physics, 120(3), 1383-1394.

This Article is brought to you for free and open access. It has been accepted for inclusion in Physics Faculty Publications and Presentations by an authorized administrator of PDXScholar. Please contact us if we can make this document more accessible: pdxscholar@pdx.edu. 


\title{
Environmental swap energy and role of configurational entropy in transfer of small molecules from water into alkanes
}

\author{
Pavel Smejtek and Robert C. Word \\ Department of Physics, Portland State University, Portland, Oregon 97207
}

(Received 4 September 2002; accepted 17 October 2003)

\begin{abstract}
We studied the effect of segmented solvent molecules on the free energy of transfer of small molecules from water into alkanes (hexane, heptane, octane, decane, dodecane, tetradecane, and hexadecane). For these alkanes we measured partition coefficients of benzene, 3-methylindole (3MI), 2,3,4,6-tetrachlorophenol (TeCP), and 2,4,6-tribromophenol (TriBP) at 3, 11, 20, 3, and $47^{\circ} \mathrm{C}$. For $3 \mathrm{MI}, \mathrm{TeCP}$, and TriBP the dependence of free energy of transfer on length of alkane chains was found to be very different from that for benzene. In contrast to benzene, the energy of transfer for 3MI, TeCP, and TriBP was independent of the number of carbons in alkanes. To interpret data, we used the classic Flory-Huggins $(\mathrm{FH})$ theory of concentrated polymer solutions for the alkane phase. For benzene, the measured dependence of energy of transfer on the number of carbons in alkanes agreed well with predictions based on FH model in which the size of alkane segments was obtained from the ratio of molar volumes of alkanes and the solute. We show that for benzene, the energy of transfer can be divided into two components, one called environmental swap energy (ESE), and one representing the contribution of configurational entropy of alkane chains. For 3MI, TeCP, and TriBP the contribution of configurational entropy was not measurable even though the magnitude of the effect predicted from the FH model for short chain alkanes was as much as 20 times greater than experimental uncertainties. From the temperature dependence of ESE we obtained enthalpy and entropy of transfer for benzene, 3MI, TeCP, and TriBP. Experimental results are discussed in terms of a thermodynamic cycle considering creation of cavity, insertion of solute, and activation of solute-medium attractive interactions. Our results suggest that correcting experimental free energy of transfer by Flory-Huggins configurational entropy term is not generally appropriate and cannot be applied indiscriminately. (c) 2004 American Institute of Physics.
\end{abstract}

[DOI: $10.1063 / 1.1633257]$

\section{INTRODUCTION}

Experimental values of free energy of transfer of small molecules from water into apolar media are important for the refinement of molecular models of solvation as well as for biomedical and nanotechnology applications. One area of interest is environmental swap energy $\left(\mathrm{ESE}_{12}\right)$, which is generally understood as the work of transfer of a solute from a fixed position in medium " 1 " into a fixed position in medium "2." In continuum and semi-empirical cavity models ESE is closely related to the solvation parameter for the hydrophobic effect. The solvation parameter, $\sigma$ or $\gamma$, relates the environmental swap energy, $\mathrm{ESE}_{12}$, or the hydration free energy $\mathrm{ESE}_{\mathrm{gw}}$ (gas $\rightarrow$ water) to either the solvent accessible surface area or solvent excluded molecular surface area, $A$, of a solute molecule ${ }^{1-4}$ according to

$$
\mathrm{ESE}_{12}=\sigma A
$$

or

$$
\mathrm{ESE}_{\mathrm{gw}}=\gamma A+b,
$$

where $b$ is the free energy of hydration of a point solute. In earlier studies, the value of the solvation parameter $\sigma$ for alkanes was found to be about $-13 \mathrm{~kJ} \mathrm{~mol}^{-1} \mathrm{~nm}^{-2}$ using mole fraction-based partition coefficients and solvent accessible surface area, and about $-20 \mathrm{~kJ} \mathrm{~mol}^{-1} \mathrm{~nm}^{-2}$ using vol- ume fraction-based partition coefficients and accounting for configurational entropy contribution using Flory-Huggins theory. ${ }^{5}$ The differences between solvation parameters illustrate the importance of concentration scales and the configurational entropy effect for the determination of ESE.

Parametrization of ESE, if possible, would greatly simplify applications of structure-activity relationships. Recent molecular modeling studies strongly suggest that predictions based on Eq. (1) are not quite correct because these relationships do not reproduce solvation free energies of aromatic, linear, branched and cyclic hydrocarbons with one set of parameters. It turns out that the free energy change associated with the cavity is related to the accessible surface area or volume of the cavity whereas the energy of solute-solvent interactions is not. This observation appears to be the origin of limitations of the applicability of Eq. (1). ${ }^{6}$ Another issue facing both experimentalists and theorists is obtaining the correct ESE. It is obvious that a molecule, charged or uncharged, when inserted into a medium causes local reorganization of the medium. In case of a medium made up of articulated molecules, such as alkanes, the presence of a solute molecule is expected to decrease the number of configurations of alkane chains surrounding the solute. This effect would alter the value of the free energy of transfer.

In this work we address the issue of the experimental 
(a)

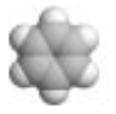

(b)

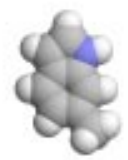

(c)

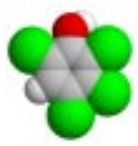

(d)

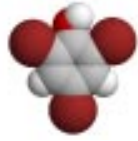

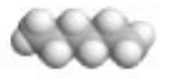
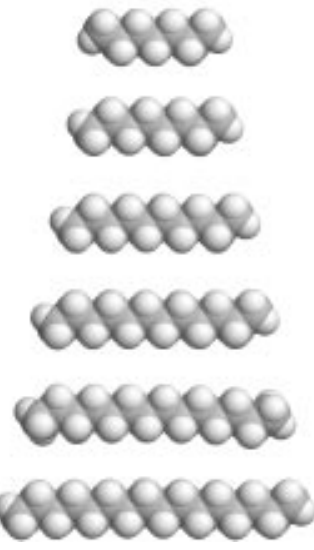

FIG. 1. Molecular structures of (a) benzene, (b) 3-methylindole, (c) 2,3,4,6tetrachlorophenol, (d) 2,4,6-tribromophenol and alkanes used in the study: hexane (C6), heptane (C7), octane (C8), decane (C10), dodecane (C12), tetradecane (C14), and hexadecane (C16).

determination of ESE and the observability of configurational entropy contribution to the free energy of solute transfer. The partition studies reported here are not conventional. In conventional partition studies, partition coefficients are typically measured for a series of solute molecules for a single pair of phases, such as water-octanol or water-lipid bilayer. Results of the present study provide information on the dependence of free energy of transfer on the number of carbons of alkanes and on the effect of temperature on the free energy of transfer. The present study includes benzene as a reference solute, and three other molecules with different degrees of "hydrophobicity:" 3-methylindole (3MI), 2,3,4,6-tetrachlorophenol ( $\mathrm{TeCP}$ ), and 2,4,6-tribromophenol (TriBP). 3MI is a side group of tryptophan and is the least "hydrophobic" of the group. Partition coefficients of 3MI have been measured for water-octanol ${ }^{7}(\log K=2.60)$ and for water-cyclohexane $^{8} \quad(\log K=1.3) . \quad$ TriBP $^{9}$ has $\log K($ water-octanol $)=4.23$. $\mathrm{TeCP}^{10}$ is the most "hydrophobic" with $\log K($ water-octanol $)=4.45$. Partition properties of 3MI are important for molecular biology and those of $\mathrm{TeCP}$ and TriBP are important for toxicology. TeCP and other halogenated phenols are known to permeate across the lipid matrix and disrupt energy conversion in biological membranes. ${ }^{11-13}$ Molecular structures of solutes and alkanes used in this study are depicted in Fig. 1.

The configurational entropy contribution to the free energy of partition of solutes, $T \Delta S_{\text {cfg }}$, is due to restriction of the number of configurations of articulated solvent molecules such as alkanes and hydrocarbon chains of lipids. The presence or absence of the configurational entropy effect is essential for the determination of environmental swap energy. This work was motivated by studies of partition of xenon from gas into alkanes, ${ }^{14,15}$ and benzene between water and alkanes. ${ }^{16}$ Ben-Naim proposed that the dependence of the free energy of transfer of Xe on the number of carbons in alkanes is due to the difference in interaction energy of Xe with methylene and methyl groups of alkanes. ${ }^{15}$ In contrast, Dill and co-workers ${ }^{16,17}$ pointed out that the difference in energy of attractive interactions between Xe and methylene and methyl groups is likely to be small, and that interpretation of Xe transfer energy based on Flory-Huggins model yields a free energy value independent of alkane length. Recently, Eads ${ }^{18}$ reanalyzed xenon partition data of Pollack and Himm $^{14}$ and Ben-Naim and Marcus ${ }^{15}$ using an improved Flory-Huggins model based on the removal of the assumption that insertion probabilities of alkane chain segments into the space lattice are uncorrelated. Eads demonstrated that one can fit the dependence of the free energy of transfer of $\mathrm{Xe}$ on the number of carbons, as well as on the temperature with the extended version of FH model. ${ }^{18}$

It is necessary to verify that concepts of solvation developed for transfer of atoms into alkanes can be applied to the transfer of molecules. It is therefore important that De Young and Dill ${ }^{16}$ showed that these concepts are applicable to the transfer of benzene between water and alkanes and that application of the FH model to measured free energies of transfer results in free energies independent of the length of alkane chains.

Since our experimental method of measuring partition coefficients is different from that used by De Young and Dill, we included benzene in our set of molecules, and performed measurements of partition coefficients for all four compounds under identical conditions. The alkanes used in our study were hexane, heptane, octane, decane, dodecane, tetradecane, and hexadecane. The temperature points were 3, $11,20,33$, and $47^{\circ} \mathrm{C}$. We have obtained the enthalpy and entropy of transfer from water into alkanes from the temperature dependence of ESE for each solute. We have shown that in the case of benzene it is possible to identify the alkane configurational entropy term $T \Delta S_{\text {cfg }}$ with the value predicted from Flory-Huggins theory. The most significant result of this study is that there is no experimental evidence for the explicit presence of $T \Delta S_{\text {cfg }}$ for the transfer of $3 \mathrm{MI}, \mathrm{TeCP}$, and TriBP between water and alkanes. Results are discussed in terms of a thermodynamic cycle involving a two-step solvation process in each phase: (1) formation of a cavity followed by (2) insertion of a solute and activation of the attractive interactions between the solute and the solvent.

\section{PARTITION OF MOLECULES AND ENVIRONMENTAL SWAP ENERGY}

The free energy of transfer of a solute from medium " 1 " into medium " 2, ," $\Delta G_{12}$, is obtained from the partition coefficient according to

$\Delta G_{12}=-R T \ln K_{p}$.

Experimental results on the partition of molecules are typically given in terms of (a) mole fraction, (b) number density or molarity, or (c) volume fraction partition coefficients. These are designated as $K_{p x}, K_{p \rho}$, and $K_{p \phi}$. The free energies of transfer determined from different concentration scales, although related, cannot be directly compared. The preferences of using a particular partition concept have been discussed. . $^{1,17,19-22}$

In simple terms, when mole fraction partition coefficients are used, it is implied that the chemical potential is 
determined by the energy of insertion of an isolated solute molecule into a given medium and the entropy contribution due to mixing. According to this approach, the chemical potential of solute molecules in media 1 and 2 are given by

$$
\begin{aligned}
& \mu_{s 1}\left(x_{1}\right)=\mu_{s 1}^{0}+R T \ln x_{1}, \\
& \mu_{s 2}\left(x_{2}\right)=\mu_{s 2}^{0}+R T \ln x_{2} .
\end{aligned}
$$

$\mu_{s 1}^{0}$ and $\mu_{s 2}^{0}$ are chemical potentials of solute molecules at infinite dilution, $x_{1}$ and $x_{2}$ are the mole fractions of solute molecules in respective media. The mole fraction-based free energy of transfer, $\Delta G_{x}$ is determined from the measured mole fraction partition coefficient $K_{p x}$

$$
\Delta G_{x}=-R T \ln K_{p x},
$$

where $K_{p x}=x_{2} / x_{1}$.

An alternative and more rigorous description of partition has been based on number densities of solute molecules, ${ }^{23} \rho$. The absolute chemical potential of solute in medium 1, $\mu_{s 1 a}$, is equal to

$$
\mu_{s 1 a}\left(\rho_{1}\right)=\mu_{s 1}^{*}+R T \ln \rho_{1} \lambda^{3},
$$

and, similarly, for medium 2

$$
\mu_{s 2 a}\left(\rho_{2}\right)=\mu_{s 2}^{*}+R T \ln \rho_{2} \lambda^{3},
$$

where $\mu_{s 1}^{*}$ and $\mu_{s 2}^{*}$ are the pseudochemical potentials of solute in media " 1 " and " 2 ;" $\rho_{1}$ and $\rho_{2}$ are the number densities of solute in " 1 " and " $2, " \lambda$ is the thermal de-Broglie wavelength of the solute,

$$
\lambda=\frac{h}{\sqrt{2 \pi m k T}} .
$$

All quantities in Eq. (6) have their generally accepted meaning. According to this approach, the environmental swap energy can be obtained from the density-based free energy of transfer, $\Delta G_{\rho}$ further identified as $\Delta G^{*}$,

$$
\Delta G^{*}=-R T \ln K_{p \rho},
$$

$K_{p \rho}=\rho_{2} / \rho_{1}$ is the number-density-based partition coefficient. The mole fraction and the density-based approaches to obtaining free energy of transfer are conceptually different ${ }^{23}$ even though the changes of $\Delta G^{*}$ for a series of solute molecules are the same as $\Delta G_{x}^{1}$.

Environmental swap energy, ESE, is in general obtained from the experimental values of $\Delta G$. Due to different concentration scales, one can obtain two different sets of ESE: the mole fraction-based $\mathrm{ESE}_{x}$ and the density-based $\mathrm{ESE}_{\rho}$.

The mole fraction-based and the density-based partition coefficients are related. In the limit of low solute concentrations

$$
K_{p \rho}=K_{p x} \frac{V_{1}^{0}}{V_{2}^{0}},
$$

where $V_{1}^{0}$ and $V_{2}^{0}$ are the molar volumes of media " 1 " and " 2 ." The low concentration requirement was met in our studies since solute concentrations were in the micromolar to millimolar range. Therefore, $K_{p \rho}$ and $K_{p x}$ are related accord- ing to Eq. (8). Consequently, the relationship between the density based and the mole fraction-based free energy of transfer is

$$
\Delta G^{*}=\Delta G_{x}-R T \ln \frac{V_{1}^{0}}{V_{2}^{0}} .
$$

Chan and Dill ${ }^{17}$ identified the above two approaches as "classical" because they do not explicitly account for the possible entropy changes in the partition media when the transfer of solute is taking place. In the literature, the entropic contribution is accounted for by an additional term $f_{c} \cdot 17$

In this work we explore, experimentally, the "observability" of the entropic term in water-to-alkane partition of 3MI, TeCP, TriBP, and benzene. Specifically, we examine the possibility that the free energy of transfer can be split into the environmental swap energy, ESE, and the configurational entropy contribution for solute in alkane, $f_{\text {cfg }}$. For the mole fraction concentration scale

$$
-R T \ln K_{p x}=\mathrm{ESE}_{x}+f_{\mathrm{cfg} x},
$$

for the number density-based partition coefficients

$$
-R T \ln K_{p \rho}=\mathrm{ESE}_{\rho}+f_{\mathrm{cfg} \rho} .
$$

There is no need to examine separately $f_{\operatorname{cfg} x}$ and $f_{\operatorname{cfg} \rho}$ because they are related to each other as

$$
f_{\operatorname{cfg} x}=f_{\operatorname{cfg} \rho}+R T \ln \frac{V_{1}^{0}}{V_{2}^{0}} .
$$

In the analysis of experimental results we adopt the density-based concentration scale since the density based $\Delta G^{*}$ has the physical content of the difference of Gibbs solvation free energies. ${ }^{23}$ The concept of Gibbs solvation free energy implies that $\Delta G^{*}$ is the environmental swap energy, ESE* $^{*}$. However, in this work, $f_{\operatorname{cfg} \rho}$ is not regarded as a correction, but as an experimentally identifiable contribution of configurational entropy to the free energy of transfer of solute from water into alkane. The physical content of $\mathrm{ESE}_{\rho}$ is the free energy of transfer, ESE* ${ }^{*}$, without the configurational entropy term due to flexible alkane chains.

On the microscopic scale, the free energy of transfer of solute molecule from water into alkane is equal to

$$
\Delta G^{*}=-R T \ln \frac{\left\langle\exp \left[-W_{\mathrm{alk}}(r) / R T\right]\right\rangle}{\left\langle\exp \left[-W_{w}(r) / R T\right]\right\rangle},
$$

where $r$ is a vector representing single configuration of $N$ medium molecules, $W(r)$ is the total potential energy of perturbation associated with the insertion of solute into the medium, brackets represent the ensemble average done over the pure-medium configurations. $W(r)$ consists of repulsive and attractive contributions.

We are concerned primarily with the experimental confirmation of the presence or absence of the configurational entropy effect in transfer of small molecules from water into alkanes. Our set of molecules is $3 \mathrm{MI}$, TeCP, TriBP, and benzene. Below we analyze the partition results in terms of the 
classic lattice model of polymer solutions discussed in 1941 by Huggins ${ }^{24}$ and Flory ${ }^{25}$ and the recent extension of the model by Eads. ${ }^{18}$

Briefly, Flory's model was developed for concentrated solutions of a linear polymer in a solvent. In our case, alkane is regarded to be a polymer and solute replaces the solvent in a treatment known as Flory-Huggins (FH) model. In the model, the alkane medium consists of $n_{\text {alk }}$ alkanes and $n_{s}$ solute molecules. The medium is viewed as a space lattice of cells of equal volume, each cell containing either a solute or a segment of alkane; since there are no empty cells, there is no free volume. The total number of cells in the lattice is $n_{s}+\nu n_{\text {alk }}$, where $\nu$ is the number of segments per one alkane molecule. In the solution, solute molecules are encapsulated by the alkanes, the number of segments is determined by the size of the solute rather than by the length of one $\mathrm{CH}_{2}$ unit. The number of segments depends on the relative size of the alkane and the solute. It follows from properties of the lattice that

$$
\nu=\frac{V_{\mathrm{alk}}^{0}}{V_{s}^{0}},
$$

where the $V_{\text {alk }}^{0}$ and $V_{s}^{0}$ are the molar volume of alkane and solute.

The total configuration entropy of mixing of $n_{\text {alk }}$ moles of straight alkane molecules with $n_{s}$ moles of solute is equal to

$$
\begin{aligned}
\Delta S_{\mathrm{tot}}= & -R\left[n_{s} \ln \left(\frac{n_{s}}{n_{s}+\nu n_{\mathrm{alk}}}\right)+n_{a} \ln \left(\frac{n_{\mathrm{alk}}}{n_{s}+\nu n_{\mathrm{alk}}}\right)\right] \\
& +R(\nu-1) n_{\mathrm{alk}}[\ln (Z-1)-1]-R n_{\mathrm{alk}} \ln 2 \\
= & \Delta S_{\mathrm{mix}}+\Delta S_{\mathrm{disor}} .
\end{aligned}
$$

The first term in the sum is the entropy of mixing the solute with disoriented alkane and the second term is the entropy of disorientation of the alkane; $Z$ is the coordination number of the cell equal to the number of nearest neighbor cells available for placing the consecutive alkane segment in the lattice. Since, in our experiments, alkanes are already disordered, we are interested in the entropy of mixing the solute with the disordered alkane. This entropy of mixing is equal to

$$
\Delta S_{\mathrm{mix}}=-R\left[n_{s} \ln \left(\frac{n_{s}}{n_{s}+\nu n_{\mathrm{alk}}}\right)+n_{\mathrm{alk}} \ln \left(\frac{n_{\mathrm{alk}}}{n_{s}+\nu n_{\mathrm{alk}}}\right)\right] .
$$

When applied to partition studies, the configurational entropy of mixing of solute with disordered alkanes is often given in terms of volume fractions,

$$
\Delta S_{\text {mix }}=-R\left[n_{s} \ln \left(\phi_{s}\right)+n_{\text {alk }} \ln \left(\phi_{\text {alk }}\right)\right],
$$

where $\phi_{s}$ and $\phi_{\text {alk }}$ are the volume fractions of solute and alkane in the mixed state, as follows from Eq. (14). $\Delta S_{\text {mix }}$ does not include any entropy contribution resulting from specific interactions between nearest neighbors. $\Delta S_{\text {mix }}$ is strictly the mixing part of the configurational entropy.
Since partition coefficients provide information on the free energy of transfer of solutes, we obtain the partial molar entropy of the solute in alkane,

$$
\begin{aligned}
\Delta S_{s}(\nu) & =\frac{\partial}{\partial n_{s}}\left(\Delta S_{\text {mix }}\right) \\
& =\frac{\partial}{\partial n_{s}}\left\{-R\left[n_{s} \ln \left(\phi_{s}\right)+n_{\text {alk }} \ln \left(\phi_{\text {alk }}\right)\right]\right\} \\
& =-R\left[\ln \phi_{s}+\left(1-\frac{1}{\nu}\right) \phi_{\text {alk }}\right] .
\end{aligned}
$$

In this study we are primarily interested in the effect of the length of alkane chains on the free energy of transfer of solute. To isolate the effect of alkane length, we obtain the excess molal entropy of solute in alkane by subtracting $\Delta S_{s}$ (mono) corresponding to alkane behaving as a nonarticulated monomer. For a nonarticulated monomer the number of segments $\nu=1$.

This excess molal entropy of solute in alkane is evaluated according to

$$
\begin{aligned}
\Delta S_{\mathrm{cfg}}= & \Delta S_{s}(\nu)-\Delta S_{s}(\mathrm{mono}) \\
= & -R\left[\ln \phi_{s}+\left(1-\frac{1}{\nu}\right) \phi_{\mathrm{alk}}\right] \\
& +R\left[\ln \phi_{s}+\left(1-\frac{1}{1}\right) \phi_{\mathrm{alk}}\right] \\
= & -R\left(1-\frac{1}{\nu}\right) \phi_{\mathrm{alk}} .
\end{aligned}
$$

The experimental conditions in our partition studies meet the requirement of Flory's theory of highly concentrated polymer solutions since the concentration of solute in alkanes is small: in the $\mu \mathrm{M}$ to $\mathrm{mM}$ range. Thus, the volume fraction of alkane in the alkane phase $\phi_{\text {alk }} \cong 1$, and the excess molal entropy of solute in alkane becomes

$$
\Delta S_{\mathrm{cfg}}=-R\left(1-\frac{1}{\nu}\right)=-R\left(1-\frac{V_{s}^{0}}{V_{\mathrm{alk}}^{0}}\right) .
$$

It follows from Eq. (18) that the longer the alkane chain, the greater the decrease of the entropy of the solute. This effect is due to the greater loss of the number of configurations of long alkane chains induced by the presence of the solute. Equation (18) also predicts the decrease in the magnitude of the configurational entropy with the increasing size of the solute.

The configurational entropy component of the free energy of transfer of solute into alkane specified by Eqs. (10a)$(10 \mathrm{c})$ is equal to

$$
f_{\mathrm{cfg} \rho}=-T \Delta S_{\mathrm{cfg}}=R T\left(1-\frac{V_{s}^{0}}{V_{\mathrm{alk}}^{0}}\right) .
$$

This equation will be used below in the analysis of experimental results. We do not consider similar contribution for the aqueous medium since water cannot be regarded as a polymer. FH model has been invoked in the literature for the 
determination of solvation parameters $\sigma$ and $\gamma$ and for corrections of free energy of transfer ${ }^{2,3,5}$ to obtain environmental swap energies.

\section{EXPERIMENTAL METHODS}

2,3,4,6-tetrachlorophenol ( $\mathrm{TeCP}$ ) was obtained from TCI America (Portland, OR). Benzene, 3-methylindole (3MI), 2,4,6-tribromophenol (TriBP), hexane, octane, decane, dodecane, tetradecane, and hexadecane were supplied by SigmaAldrich (St. Louis, MO). Heptane was obtained from B\&J, Inc. (Muskegon, MI). These compounds were used as supplied.

Partition coefficients were determined using the traditional "shake-flask" method. ${ }^{26}$ In our procedure $25.00 \mathrm{~mL}$ of a pH 3.0 potassium borate phosphate buffer $\left(2.0 \mathrm{mM} \mathrm{KPO}_{4}\right.$, $2.0 \mathrm{mM} \mathrm{H}_{3} \mathrm{BO}_{3}$, and $0.030 \mathrm{M} \mathrm{KCl}$ ) and $5.00 \mathrm{~mL}$ of an alkane solution were added to $50-\mathrm{mL}$ glass centrifuge tubes (VWR, Inc., West Chester, PA). The initial concentrations of 3MI, TeCP, and TriBP in alkanes were between 3 and 20 $\mathrm{mM}$. The initial concentration of benzene in alkanes was $56.3 \mathrm{mM}$. Because solutions were prepared at room temperature $\left(20^{\circ} \mathrm{C}\right)$ volumes and concentrations were adjusted in our calculations to account for thermal expansion of alkanes. The flasks were each shaken sharply 10 times by hand and placed in a water bath for 20 hours at a constant temperature of 3.0, 11.0, 20.0, 33.0, or $47.0^{\circ} \mathrm{C}\left( \pm 0.1{ }^{\circ} \mathrm{C}\right)$. The following day the flasks were removed from the bath. The alkane phases were extracted by pipette and discarded. The equilibrium concentration of the solute was determined from measurement of the UV absorbance of water phases using a Beckman DU-7 spectrophotometer (Irvine, CA). TeCP and TriBP aqueous solutions were mixed with $0.1 \mathrm{M} \mathrm{KOH} \mathrm{(4:1}$ volume ratio) to adjust their $\mathrm{pH}$ to about 12 so that the absorbance of the ionized species was measured. This was done because the higher solubility of the ionized species makes its extinction coefficient easier to determine. The equilibrium concentrations of the solutes in alkane were not measured directly, but were calculated using the mass balance equation

$$
[\text { solute }]_{f, \text { alk }}=[\text { solute }]_{i, \text { alk }}-\frac{V_{w}}{V_{\mathrm{alk}}}[\text { solute }]_{f, w} .
$$

The subscripts $i$ and $f$ refer to initial and final concentrations. $V_{w}$ and $V_{\text {alk }}$ are the respective volumes of the water and alkane phases.

Partition coefficients were measured from three to nine times for each set of experimental conditions. The mean standard deviations of the partition coefficients were $2.8 \%$ for $3 \mathrm{MI}, 5.4 \%$ for benzene, $4.8 \%$ for $\mathrm{TeCP}$, and $6.5 \%$ for TriBP.

\section{RESULTS}

\section{A. Benzene}

We have measured density-based partition coefficients of benzene between water and alkanes at temperatures, 3, 11, 20,33 , and $47^{\circ} \mathrm{C}$ and obtained the free energy of transfer $\Delta G^{*}$ according to Eq. (7). The experimental free energies are shown in Fig. 2(a). The two major features of the results are that (1) $\Delta G^{*}$ become more negative with the increasing temperature for all alkanes and (2) for a constant temperature $\Delta G^{*}$ becomes more positive with the increasing length of alkane chain. The latter effect indicates that the probability of transfer of benzene from water into alkane decreases with the length of alkane chain. At 3 and $11^{\circ} \mathrm{C}$ the alkane sequences shown in Fig. 2(a) are incomplete because longer alkanes are not liquids at these temperatures.

Configurational entropy effect: The free energies of transfer were analyzed in terms of Eq. (10b) where the configurational entropy term $f_{\text {cfg } \rho}$ was either determined by Eq. (19) (FH model) or by Eads' lattice, Eq. (42) in Ref. 18. Eads' model accounts more accurately for the statistics of shorter alkane chains than the Flory-Huggins model. We found that both models explained the alkane length and the temperature dependence of the measured free energies of transfer. The solid curves in Fig. 2(a) illustrate the LSF fit of FH model to the measured data obtained from the combination of Eqs. (10b) and (19). Due to the incomplete alkane sequencies at 3 and $11{ }^{\circ} \mathrm{C}$, the overall agreement between model predictions and data was even better with the simpler FH model.

Environmental swap energy, $\mathrm{ESE}_{\rho}$, at each temperature, was obtained from the fit of the model to the data on dependence of free energy of transfer as a function of the number of carbons in alkane. The plot of the temperature dependence of $\mathrm{ESE}_{\rho}$ is shown in Fig. 2(b). The dependence is linear with $r^{2}=0.995$ so it is therefore possible to obtain enthalpic and entropic components of $\mathrm{ESE}_{\rho}$. These quantities are listed in Table I. The linearity of $\mathrm{ESE}_{\rho}$ vs $T$ plot indicates that presence of heat capacity of transfer, $\Delta C_{p}$, does not have to be invoked.

Figure 2(c) depicts the magnitude of configurational entropy contribution for benzene as a function of alkane length and temperature. The alkane dependence of $f_{\operatorname{cfg} \rho}$ for benzene is important in the analysis of conditions of observability of configurational entropy effect below.

The properties of $\operatorname{ESE}_{\rho}$ are listed in Table I. The data indicate (1) that the potential energy of benzene in water is slightly lower than that in alkanes since $\Delta H_{0}>0$, (2) that the entropy of benzene and the system increases on swapping the aqueous environment of benzene by alkane, and (3) that energetics of transfer is dominated by the change of entropy, viz. $-T \Delta S_{0} / \Delta H_{0} \approx 24$, which is typical of the conventional hydrophobic effect. The applicability of mean-field FH model to transfer of benzene into alkane of variable length with one value of interaction energy is in agreement with the assumption that interactions between benzene and methylene and methyl groups of alkanes are about the same, as anticipated by Chan and Dill. ${ }^{17}$

The free energy of transfer of benzene obtained in this study and our conclusion that it is possible to identify the configurational entropy contribution to the free energy of transfer are in agreement with results of earlier study of De Young and Dill. ${ }^{16}$ The agreement is significant because in our studies we have used a different experimental method of determination of water-to-alkane partition coefficients. 
(a)

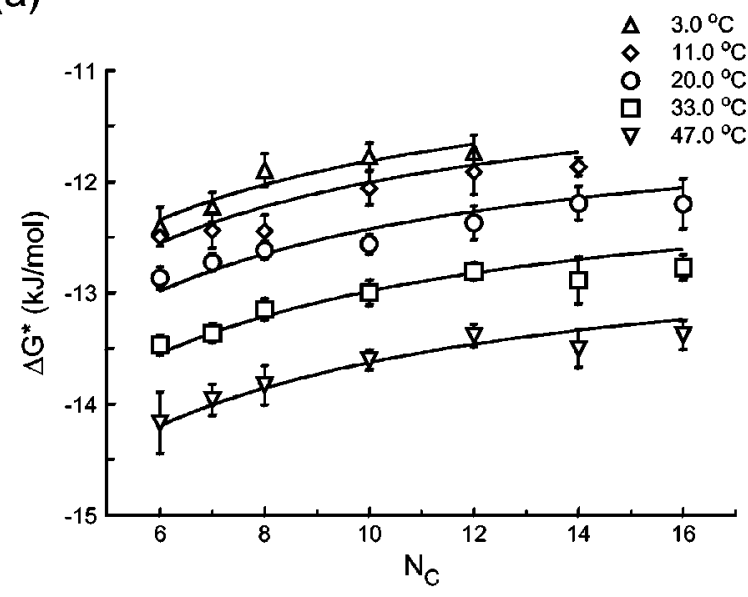

(b)

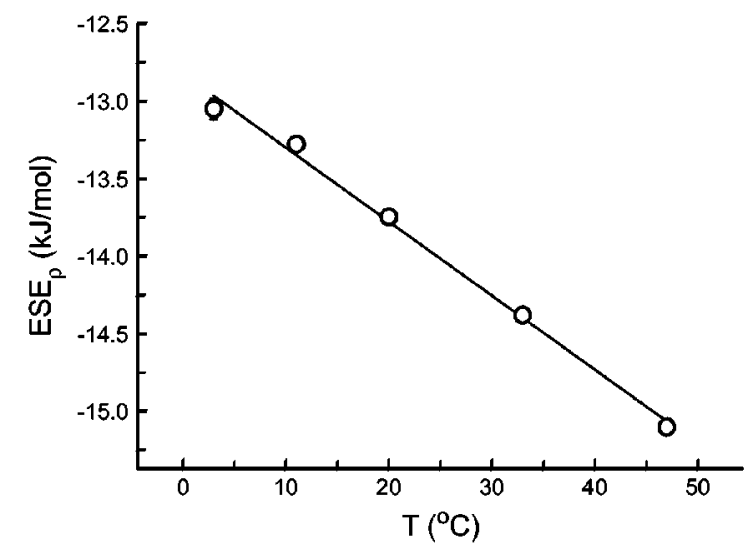

(c)

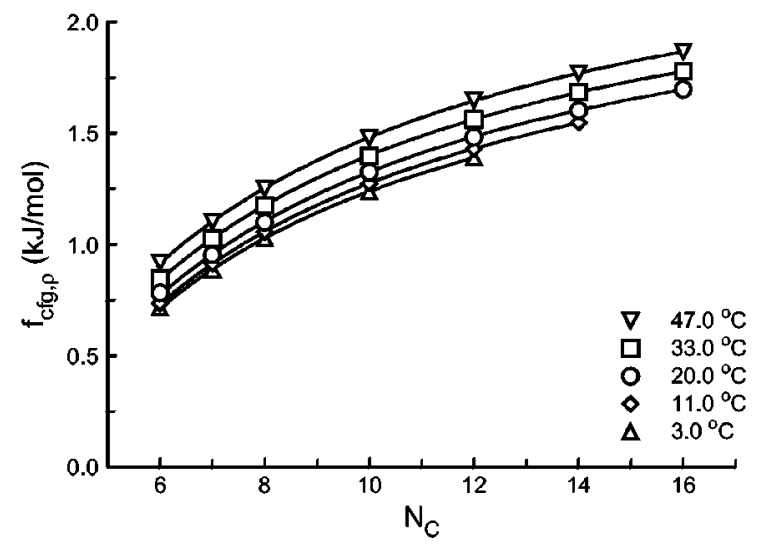

FIG. 2. (a) Benzene. Experimental values of the density-based free energy of transfer from water into alkanes as a function of temperature and the number of alkane carbon atoms. The solid curves illustrate the least-squares fit of FH model based on Eqs. (10b) and (19). (b) Benzene. Temperature dependence of environmental swap energy, $\mathrm{ESE}_{\rho}$, obtained from the leastsquares fit of FH model to the experimental data shown in (a), $r^{2}=0.995$. Linearity of the plot indicates that it is meaningful to split the free energy of transfer according to Eq. (10b). (c) Benzene. Configurational entropy component, $f_{\mathrm{cfg}, \rho}$, of the free energy of transfer used in the fit of experimental results in (a). The dependence of configurational entropy term on the number of carbons in alkane chain and temperature was obtained according to Eq. (19).
TABLE I. Properties of water-to-alkane environmental swap energy, $\mathrm{ESE}_{\rho}$, for benzene.

\begin{tabular}{cc}
\hline \hline$\Delta S_{0}\left(\mathrm{~J} \mathrm{~mol}^{-1} \mathrm{~K}^{-1}\right)$ & $48.9 \pm 1.4$ \\
$\Delta H_{0}\left(\mathrm{~kJ} \mathrm{~mol}^{-1}\right)$ & $+0.60 \pm 0.40$ \\
$\Delta G_{\rho}$ at $20^{\circ} \mathrm{C}\left(\mathrm{kJ} \mathrm{mol}^{-1}\right)$ & $-13.75 \pm 0.04$ \\
$-T \Delta S_{0}$ at $20^{\circ} \mathrm{C}\left(\mathrm{kJ} \mathrm{mol}^{-1}\right)$ & $-14.3 \pm 0.4$ \\
\hline \hline
\end{tabular}

\section{B. 3-methylindole, 2,3,4,6-tetrachlorophenol, 2,4,6-tribromophenol}

Experimental data on the effect of temperature and the length of alkane chain are shown in Figs. 3(a), 4(a), and 5(a) for $3 \mathrm{MI}, \mathrm{TeCP}$, and TriBP, respectively. The dependence of energy of transfer on alkane length is qualitatively different from that of benzene, viz. the free energy of transfer does not become more positive with the increasing length of alkane chain as it was observed for benzene [Fig. 2(a)].

Next we address the issue of the magnitude and the conditions of observability of configurational entropy contribution. The alkane chain dependences of the expected contribution of configurational entropy term to the free energy of transfer are shown in Figs. 3(b), 4(b), and 5(b). They were computed according to Eq. (19) using the molar volumes of solutes and alkanes in a similar way as those for benzene. It further follows from the comparison of experimental uncertainties and the magnitude of expected configurational entropy contribution that the effect observed for benzene should be also observable for $3 \mathrm{MI}$, TeCP, and TriBP. The standard deviations of individual data sets are typically $0.1-$ $0.2 \mathrm{~kJ} / \mathrm{mol}$ whereas the magnitude of the expected configurational entropy contribution is $\approx 1.0-1.5 \mathrm{~kJ} / \mathrm{mol}$, depending on the solute. Thus the effect of alkane chain length on transfer of $3 \mathrm{MI}, \mathrm{TeCP}$, and TriBP would be measurable, if present.

The data in Figs. 3(a), 4(a), and 5(a) also suggest that there is no dominant trend of the dependence of free energy of transfer on alkane chain length. We can therefore conclude that according to Eq. (10b), the free energy of transfer for $3 \mathrm{MI}, \mathrm{TeCP}$, and TriBP is equal to the environmental swap energy. The absence of a dominant trend of the environmental swap energy on the ratio of the number of methylene groups to methyl groups also indicates that the interaction energy between $3 \mathrm{MI}, \mathrm{TeCP}$, and TriBP on the one hand, and methylene and methyl groups on the other hand, are about the same. In this study the ratio the number of methylene and methyl groups changed from 2 for hexane to 7 in hexadecane.

To gain insight into enthalpic and entropic contributions to environmental swap energy we obtain, for each temperature, a representative value of ESE and its standard deviation by averaging ESE values over the alkane chain length. The averaged ESEs are temperature dependent. Their dependence on temperature is shown in Figs. 3(c), 4(c), and 5(c) where the straight lines illustrate the linear LSF. The enthalpy and entropy as well as other thermodynamic quantities are summarized in Table II.

For 3MI, which is the least "hydrophobic" compound in our set, the enthalpic and entropic contributions to environmental swap energy are comparable, at $20^{\circ} \mathrm{C}$ the value of $T \Delta S_{0} / \Delta H_{0} \approx 1.6$. On the other hand, the entropic contribu- 
(a)

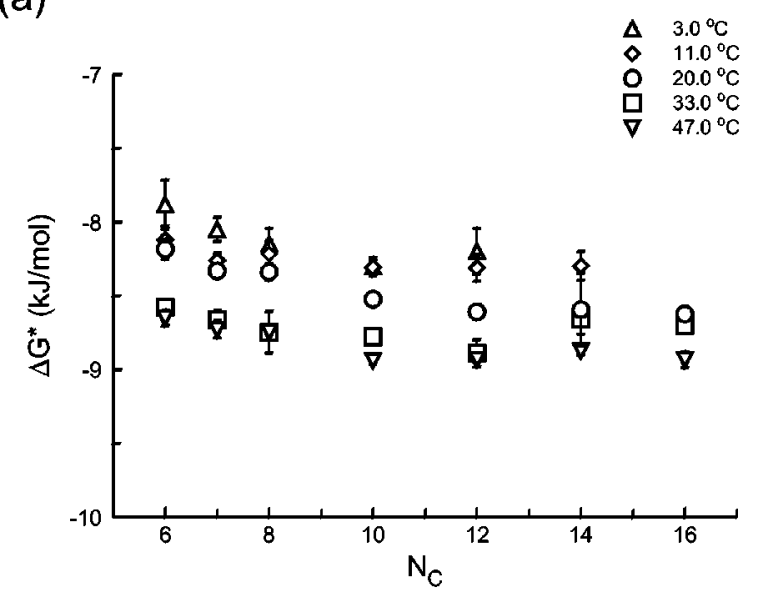

(b)

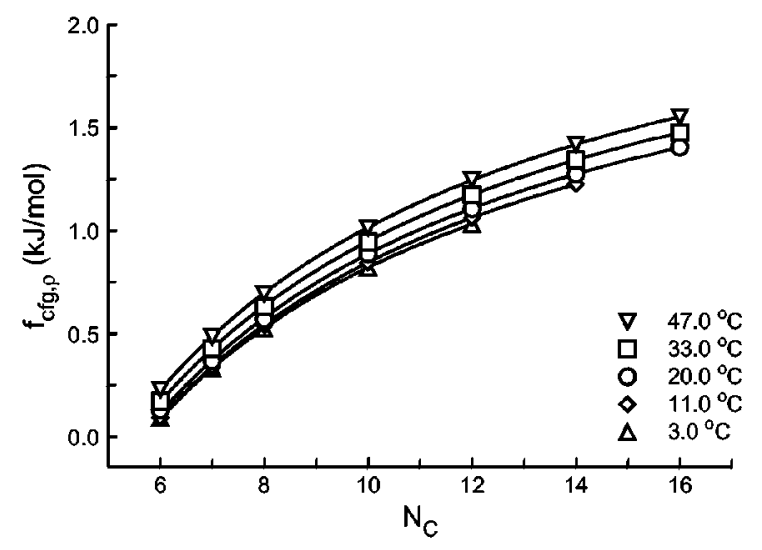

(c)

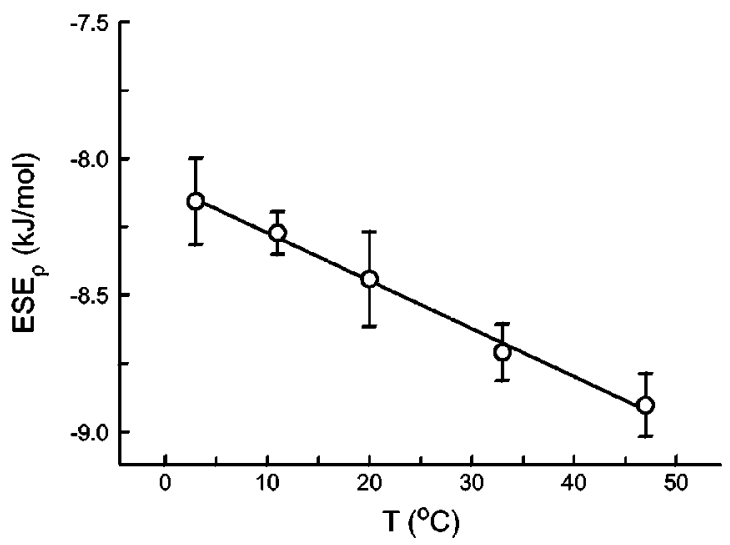

FIG. 3. (a) 3-methylindole. Experimental values of the density-based free energy of transfer from water into alkanes as a function of alkane length and temperature. In contrast to benzene, the free energy of transfer does not become more positive with increasing alkane length. (b) 3-methylindole. The expected dependence of configurational entropy contribution to the free energy of transfer on the length of alkane chain. Values of $f_{\text {cfg } \rho}$ were obtained from Flory-Huggins model using Eq. (20), i.e., the same way as for benzene. (c) 3-methylindole. Temperature dependence of environmental swap energy, $\mathrm{ESE}_{\rho}$, obtained from the least-squares fit of $\mathrm{FH}$ model to the experimental data shown in (a). The plot is linear with $r^{2}=0.995$. Thermodynamic quantities in Table II were obtained from the linear fit. (a)

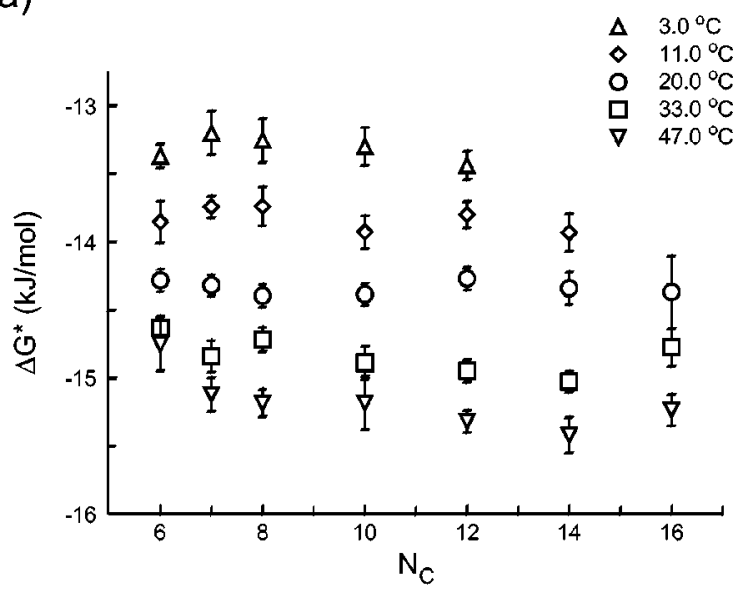

(b)

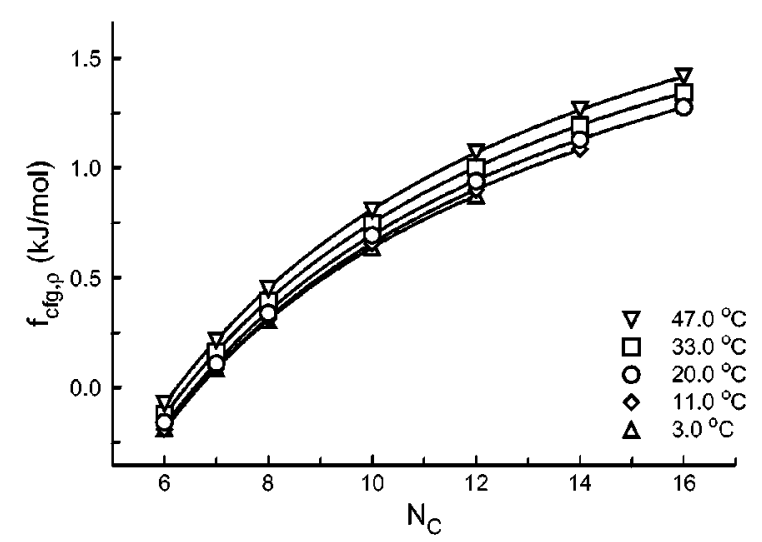

(c)

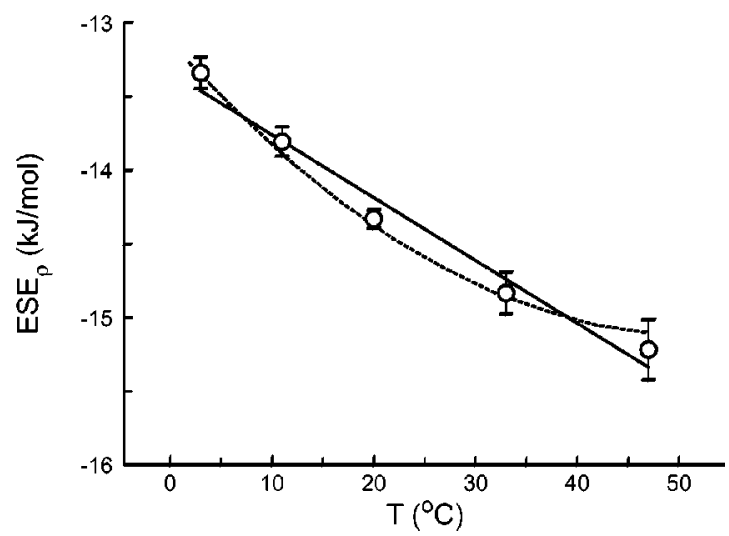

FIG. 4. (a) 2,3,4,6-tetrachlorophenol. Experimental values of density-based free energy of transfer from water into alkanes as a function of alkane length and temperature. In contrast to benzene, the free energy of transfer of TeCP does not become more positive with increasing alkane length. (b) 2,3,4,6tetrachlorophenol. The expected dependence of configurational entropy contribution to the free energy of transfer on the alkane length. Values of $f_{\operatorname{cfg} \rho}$ were obtained from Flory-Huggins model using Eq. (20), i.e., the same way as those for benzene. (c) 2,3,4,6-tetrachlorophenol. Temperature dependence of environmental swap energy, $\operatorname{ESE}_{\rho}$, obtained from the least-squares fit of FH model to the experimental data shown in (a). The plot is linear with $r^{2}=0.975$. Thermodynamic quantities in Table II were obtained from the linear fit. The broken curve illustrates the fit using constant heat capacity $\Delta C_{p}=-0.448 \mathrm{~kJ} / \mathrm{mol} \mathrm{K}$ and $\Delta S_{0}=45.7 \mathrm{~J} / \mathrm{mol} \mathrm{K}, \Delta H_{0}=-0.99 \mathrm{~kJ} / \mathrm{mol}$ at $T=294 \mathrm{~K}$. 
(a)

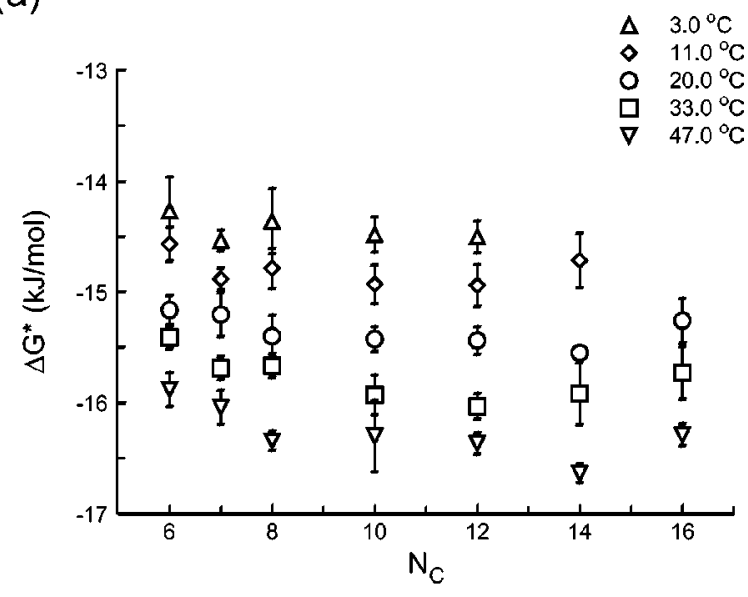

(b)

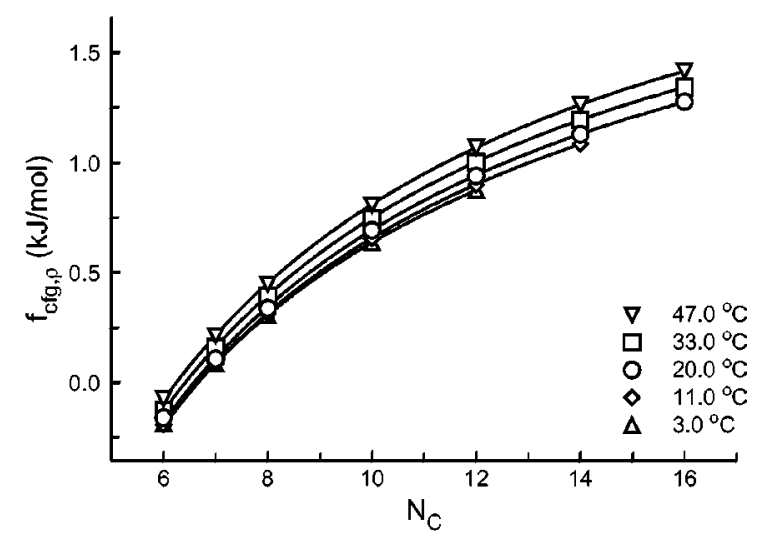

(c)

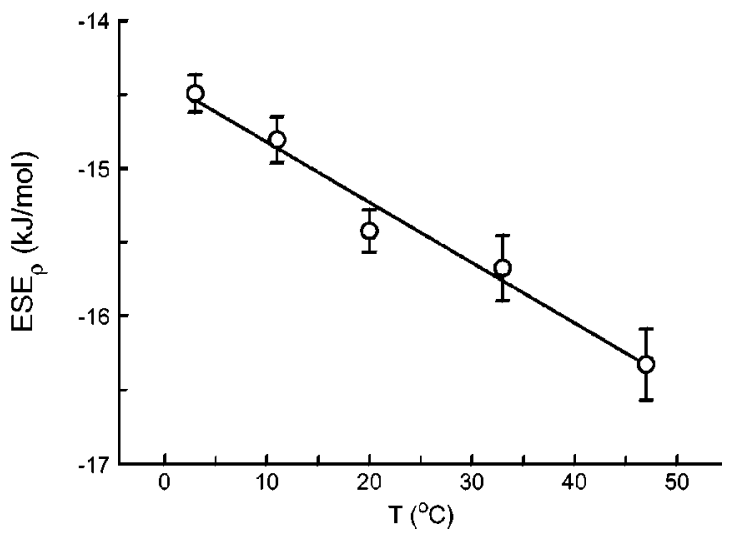

FIG. 5. (a) 2,4,6-tribromophenol. Experimental values of density-based free energy of transfer from water into alkanes as a function of alkane length and temperature. In contrast to benzene, the free energy of transfer does not become more positive with increasing alkane length. (b) 2,4,6tribromophenol. The expected dependence of configurational entropy contribution to the free energy of transfer on the length of alkane chain. Values of $f_{\text {cfgo }}$ were obtained from Flory-Huggins model using Eq. (20), i.e., the same way as for benzene. (c) 2,4,6-tribromophenol. Temperature dependence of environmental swap energy, $\mathrm{ESE}_{\rho}$, obtained from the least-squares fit of FH model to the experimental data shown in (a). The plot is linear with $r^{2}=0.976$. Thermodynamic quantities in Table II were obtained from the linear fit.
TABLE II. Properties of water-to-alkane environmental swap energy, $\mathrm{ESE}_{\rho}$, for 3-methylindole, 2,3,4,6-tetrachlorophenol, and 2,4,6-tribromophenol based on linear LSF.

\begin{tabular}{cc}
\hline \hline & \\
$\Delta H_{0}\left(\mathrm{~kJ} \mathrm{~mol}^{-1}\right)$ & $-3.2 \pm 0.97$ \\
$\Delta S_{0}\left(\mathrm{~J} \mathrm{~mol}^{-1} \mathrm{~K}^{-1}\right)$ & $17.9 \pm 3.3$ \\
$\Delta G_{\rho}$ at $20^{\circ} \mathrm{C}\left(\mathrm{kJ} \mathrm{mol}^{-1}\right)$ & $-8.44 \pm 0.16$ \\
$-T \Delta S_{0}$ at $20^{\circ} \mathrm{C}\left(\mathrm{kJ} \mathrm{mol}^{-1}\right)$ & $-5.24 \pm 0.96$ \\
$T \Delta S_{0} / \Delta H_{0}$ at $20^{\circ} \mathrm{C}$ & $1.64 \pm 0.58$ \\
$2,3,4,6$-tetrachlorophenol & \\
$\Delta H_{0}\left(\mathrm{~kJ} \mathrm{~mol}^{-1}\right)$ & $-0.45 \pm 1.24^{\mathrm{a}}$ \\
$\Delta S_{0}\left(\mathrm{~J} \mathrm{~mol}^{-1} \mathrm{~K}^{-1}\right)$ & $47.1 \pm 4.2^{\mathrm{a}}$ \\
$\Delta G_{\rho}$ at $20^{\circ} \mathrm{C}\left(\mathrm{kJ} \mathrm{mol}^{-1}\right)$ & $-14.33 \pm 0.06$ \\
$-T \Delta S_{0}$ at $20^{\circ} \mathrm{C}\left(\mathrm{kJ} \mathrm{mol}^{-1}\right)$ & $-13.8 \pm 1.2$ \\
$T \Delta S_{0} / \Delta H_{0}$ at $20^{\circ} \mathrm{C}$ & $\mathrm{N} / \mathrm{A}$ \\
& \\
$\Delta H_{0}\left(\mathrm{~kJ} \mathrm{~mol}^{-1}\right)$ & $-3.01 \pm 1.55$ \\
$\Delta S_{0}\left(\mathrm{~J} \mathrm{~mol}{ }^{-1} \mathrm{~K}^{-1}\right)$ & $41.6 \pm 5.4$ \\
$\Delta G_{\rho}$ at $20^{\circ} \mathrm{C}\left(\mathrm{kJ} \mathrm{mol}^{-1}\right)$ & $-15.34 \pm 0.17$ \\
$-T \Delta S_{0}$ at $20^{\circ} \mathrm{C}\left(\mathrm{kJ} \mathrm{mol}^{-1}\right)$ & $-12.2 \pm 1.57$ \\
$T \Delta S_{0} / \Delta H_{0}$ at $20^{\circ} \mathrm{C}$ & $4.05 \pm 2.2$ \\
\hline
\end{tabular}

${ }^{\mathrm{a}}$ The linear LSF ignores the curvature of $\mathrm{TeCP}$ data.

tion clearly dominates the environmental swap energy of $\mathrm{TeCP}$ and TriBP (Table II). In spite of the presence of an $-\mathrm{OH}$ group and chlorine or bromine substituents on the benzene ring, as in $\mathrm{TeCP}$ and TriBP, the entropy change associated with the swap of water by alkane is about the same as that of benzene (Table I). This agreement suggests that the entropy change is primarily determined by the closure of the cavity in water on the transfer of the solute into alkane.

In the above discussion, we ignored the curvature of the temperature dependence of TeCP data [Fig. 4(c)]. Below we show that the curvature can be accounted for by molar heat capacity change $\Delta C_{p}$ associated with the transfer from water into alkane. The broken curve in Fig. 4(c) illustrates the least squares fit assuming constant heat capacity $\Delta C_{p}$ $=-0.448 \mathrm{~kJ} / \mathrm{mol} \mathrm{K}$ and $\Delta S_{0}=45.7 \mathrm{~J} / \mathrm{mol} \mathrm{K}, \quad \Delta H_{0}$ $=-0.99 \mathrm{~kJ} / \mathrm{mol}$ at $T=294 \mathrm{~K}$. There is no evidence for significant $\Delta C_{p}$ in $3 \mathrm{MI}$ and TriBP data.

\section{DISCUSSION AND CONCLUSIONS}

This work was motivated by the problem of observability of the effect of segmented media on the energetics of transfer of small molecules from water into alkanes and acyl chains of lipid bilayers. We have measured water-to-alkane partition coefficients of four small hydrophobic molecules: $3 \mathrm{MI}, \mathrm{TeCP}$, and TriBP and benzene and obtained the free energy of transfer as a function of length of alkane chain $(\mathrm{C} 6-\mathrm{C} 16)$ and temperature $\left(3-47^{\circ} \mathrm{C}\right)$. Benzene was used as a reference compound since De Young and Dill ${ }^{16}$ have shown that the free energy of transfer of benzene can be made independent of alkane chain length using FH theory. Our experimental method was however different from the method employed by De Young and Dill ${ }^{16}$ so we used benzene as a control. We have found that the experimental results from both studies were similar and therefore the differences between results for benzene and $3 \mathrm{MI}, \mathrm{TeCP}$, and TriBP cannot be associated with differences between methods. 
Studies of partition of 3MI, TeCP, and TriBP were done under the same set of conditions as those for benzene.

We found the dependence of energy of transfer on length of alkane chains for $3 \mathrm{MI}, \mathrm{TeCP}$, and TriBP was very different from that of benzene. In contrast to benzene, the energy of transfer for 3MI, TeCP, and TriBP was found to be independent of the number of carbons in alkanes.

To model the alkane phase, we used Flory's classic model for concentrated solutions of polymers. We have shown that the free energy of transfer of benzene from water into alkanes of variable length consists of two contributions: one identified as environmental swap energy - a concept pioneered by Dill and co-workers, and the second one representing the configurational entropy contribution associated with flexible alkane chains. For benzene, we found excellent agreement between the predictions of $\mathrm{FH}$ model and the measured dependence of energy of transfer on number of carbons in alkanes. It was possible to determine the enthalpy and entropy of transfer of benzene from the temperature dependence of environmental swap energy.

FH model was expected to be applicable to other molecules such 3MI, TeCP, and TriBP. We have shown by comparing the magnitude of the alkane configurational entropy effect predicted from FH model with the experimental uncertainties of the free energy of transfer for $3 \mathrm{MI}, \mathrm{TeCP}$, and TriBP that the configurational entropy effect should be easily observable for these molecules as well. Instead, the experimental results indicate that the transfer energy for 3MI, TeCP, and TriBP is independent of alkane length.

Although the free energy of transfer in the FH model consists of contact terms and the configurational entropy term, it is useful to discuss the transfer process, and the experimental results, in terms of the more recent concepts of hydrophobicity and solvation. In Fig. 6 we depict thermodynamic cycle used in the discussion of the processes of solvation and transfer of molecules in our study. Since enthalpy and entropy are functions of state, the path of arriving at any given state does not matter.

It is further assumed that solvation of solute in water and alkanes can be accomplished in two steps: creation of empty cavity followed by insertion of solute and turning on attractive interactions. Adopting the solvation processes discussed in work of Graziano ${ }^{27}$ and Gallicchio, Kubo, and Levy ${ }^{6}$ and starting from creation of cavity in water, there is a reorganization of hydrogen bonds in the process, $r \# 1$. This process has enthalpy change, $\Delta H_{c, w}^{r \# 1}$ associated with it. The configurational space for water's hydrogen bonds decreases because space becomes occupied by the cavity. This leads to an entropy change for the cavity equal to $\Delta S_{\mathrm{cfg}, w}$. This decrease of entropy is proportional to the volume of displaced water. ${ }^{28}$ The proportionality of $\Delta S_{\mathrm{cfg}, w}$ to cavity volume is applicable to solutes with radius below $1 \mathrm{~nm}$. This condition is met for benzene, 3MI, TeCP, and TriBP. Due to entropy-enthalpy compensation associated with reorganization $r \# 1$, there is additional entropy change $\Delta H_{c, w}^{r \# 1} / T$.

The net free energy change due to creation of a cavity in water is equal to

$$
\Delta G_{c, w}=-T \Delta S_{\mathrm{cfg}, w} .
$$

\section{Creation of Cavity in Alkane}

Insertion of Solute into Cavity Activation of Solute-Alkane Interactions

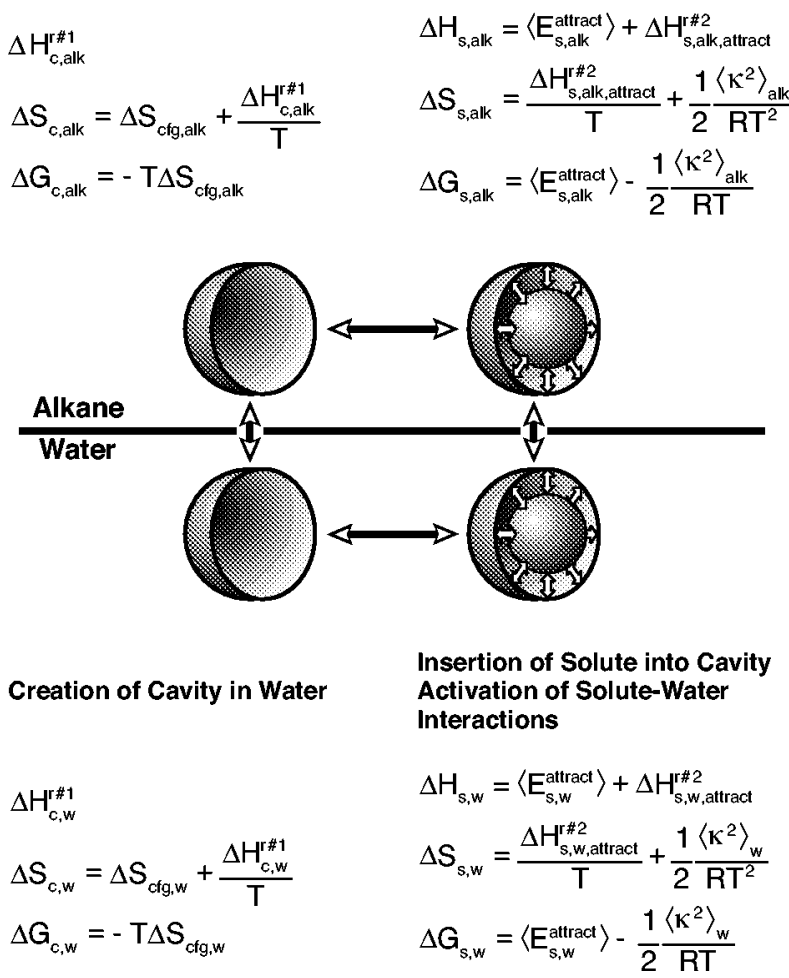

FIG. 6. Thermodynamic cycle depicting the transfer processes assuming that solvation in each phase is taking place in two steps: (1) formation of an empty cavity and (2) insertion of a solute into the cavity followed by activation of solute-medium interaction. There is reorganization of water or alkane phases, $r \# 1$, associated with the formation of the cavity, and reorganization, $r \# 2$, accompanying attractive interactions. Configurational entropy of hydrogen bonds dominates the formation of the cavity in water. Configurational entropy of alkane chains is assumed to be associated with the formation of cavity in alkanes.

The insertion of a solute into an empty cavity and the subsequent turning-on of attractive interactions between solute and water results in enthalpy that is primarily due to the change of the potential energy of the solute. The change of potential energy of the solute is equal to the average energy of solute-water interactions, $\left\langle E_{s, w}^{\text {attr }}\right\rangle$. An additional enthalpy change, $\Delta H_{s, w \text {,attract }}^{r \# 2}$, is associated with reorganization $r \# 2$. The entropy change is in part due to entropy-enthalpy compensation of reorganization of water, $r \# 2$, in the shell surrounding the solute, $\Delta H_{s, w}^{r \# 2}$,attract $/ T$, and the entropy change associated with fluctuation of solute-water interaction, $-\left\langle\kappa_{s}^{2}\right\rangle_{w} / 2 R T^{2}$.

The energy of attractive interactions between the solute and any acceptable configuration of molecules of medium, $m$, containing the cavity, and specified by configuration vector $r$, is equal to the average energy, $\bar{E}_{s, m}^{\text {attract }}$, and the deviation from the average, $\kappa_{s, m}$,

$$
W_{s, m}^{\text {attract }}(r)=\bar{E}_{s, m}^{\text {attract }}+\kappa_{s, m}(r) .
$$

The averaged contribution of fluctuations is $-\left\langle\kappa_{s}^{2}\right\rangle_{m} / 2 R T$. The free energy associated with solute-water attractive interactions is equal to 


$$
\Delta G_{s, w}=\left\langle E_{s, w}^{\mathrm{attract}}\right\rangle-\frac{1}{2} \frac{\left\langle\kappa_{s}^{2}\right\rangle_{w}}{R T} .
$$

In summary, the total solvation free energy in water has two components, a contribution from cavity formation and a contribution from solute-water attractive interactions

$$
\Delta G_{\mathrm{tot}, w}=\left\langle E_{s, w}^{\mathrm{attract}}\right\rangle-T \Delta S_{\mathrm{cfg}, w}-\frac{1}{2} \frac{\left\langle\kappa_{s}^{2}\right\rangle_{w}}{R T} .
$$

In an alkane phase the solvation steps are assumed to be similar. Creation of a cavity in alkane results is an enthalpy change due to the reorganization of alkane chains, $r \# 1$, equal to $\Delta H_{c \text {, alk }}^{r \# 1}$, and entropy change, $\Delta S_{c \text {, alk }}$. The entropy change associated with the creation of a cavity in alkane consists of the configurational contribution, $\Delta S_{\mathrm{cfg} \text {, alk }}$, plus the enthalpy compensated term $\Delta H_{c, \text { alk }}^{r \# 1} / T$. The net free energy change due to creation of a cavity in alkane is determined by the configurational entropy,

$$
\Delta G_{c, \text { alk }}=-T \Delta S_{\mathrm{cfg}, \mathrm{alk}} .
$$

The insertion of a solute into a cavity and the turning on of attractive solute-alkane interactions results in an enthalpy change consisting of the shift of the potential energy of the solute. The enthalpy change is equal to the average attractive potential energy $\left\langle E_{s, \text { alk }}^{\text {attr }}\right\rangle$, and an enthalpy contribution from the reorganization $r \# 2, \Delta H_{s \text {, alk, attract }}^{r \# 2}$ The entropy change caused by the presence of a solute in the cavity consists of the enthalpy compensated term due to reorganization $r \# 2$,

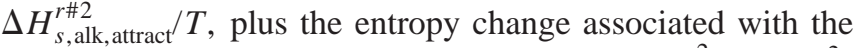
fluctuations of solute-alkane interactions, $-\left\langle\kappa_{s}^{2}\right\rangle_{\mathrm{alk}} / 2 R T^{2}$. The free energy change assigned to solute-alkane attractive interactions is

$$
\Delta G_{s, \text { alk }}=\left\langle E_{s, \text { alk }}^{\text {attract }}\right\rangle-\frac{1}{2} \frac{\left\langle\kappa_{s}^{2}\right\rangle_{\mathrm{alk}}}{R T}
$$

resulting in the net free energy change on solvation in alkane equal to

$$
\Delta G_{\mathrm{tot}, \mathrm{alk}}=\left\langle E_{s, \text { alk }}^{\mathrm{attract}}\right\rangle-T \Delta S_{\mathrm{cfg}, \mathrm{alk}}-\frac{1}{2} \frac{\left\langle\kappa_{s}^{2}\right\rangle_{\mathrm{alk}}}{R T} .
$$

It then follows that the free energy of transfer of solute from water into alkane, $\Delta G^{*}$, is

$$
\begin{aligned}
\Delta G^{*}= & \left\langle E_{s, \text { alk }}^{\text {attract }}\right\rangle-T \Delta S_{\mathrm{cfg}, \text { alk }}-\frac{1}{2} \frac{\left\langle\kappa_{s}^{2}\right\rangle_{\mathrm{alk}}}{R T}-\left\langle\left\langle E_{s, w}^{\mathrm{attract}}\right\rangle\right. \\
& \left.-T \Delta S_{\mathrm{cfg}, w}-\frac{1}{2} \frac{\left\langle\kappa_{s}^{2}\right\rangle_{w}}{R T}\right) \\
= & \left\langle E_{s, \text { alk }}^{\mathrm{attract}}\right\rangle-\left\langle E_{s, w}^{\mathrm{attract}}\right\rangle-\frac{1}{2} \frac{\left\langle\kappa_{s}^{2}\right\rangle_{\mathrm{alk}}-\left\langle\kappa_{s}^{2}\right\rangle_{w}}{R T} \\
& -T\left(\Delta S_{\mathrm{cfg}, \text { alk }}-\Delta S_{\mathrm{cfg}, w}\right) .
\end{aligned}
$$

In our study we have explored the observability of configurational entropy contribution of alkanes since it depends, in general, on the length of alkane chains. We have used, as a working hypothesis that the free energy of transfer can be split into two terms [refer to Eq. (10b)],

$$
\Delta G^{*}=\mathrm{ESE}_{\rho}+f_{\mathrm{cfg} \rho},
$$

the environmental swap energy and the configurational entropy contribution of alkane chains. In view of the above cavity model, the environmental swap energy is determined by the difference of energy of attractive interactions, the difference in energy fluctuation terms, and the configurational entropy term of hydrogen bonds in water associated with the existence of cavity in water,

$$
\begin{aligned}
\operatorname{ESE}_{\rho}= & \left\langle E_{s, \text { alk }}^{\mathrm{attract}}\right\rangle-\left\langle E_{s, w}^{\mathrm{attract}}\right\rangle-\frac{1}{2} \frac{\left\langle\kappa_{s}^{2}\right\rangle_{\mathrm{alk}}-\left\langle\kappa_{s}^{2}\right\rangle_{w}}{R T} \\
& +T \Delta S_{\mathrm{cfg}, w} .
\end{aligned}
$$

The additional contribution, $f_{\operatorname{cfg} \rho}$, is configurational entropy term associated with alkane chains - a term present for benzene and absent for $3 \mathrm{MI}, \mathrm{TeCP}$, and TriBP.

From the temperature dependence of measured environmental swap energy we obtained enthalpic and entropic contributions,

$$
\mathrm{ESE}_{\rho}=\Delta H_{0}-T \Delta S_{0} .
$$

The experimental values are summarized in Tables I and II.

Thus the physical meaning of the enthalpic component of ESE is

$$
\Delta H_{0}=\left\langle E_{s, \text { alk }}^{\text {attract }}\right\rangle-\left\langle E_{s, w}^{\mathrm{attract}}\right\rangle-\frac{1}{2} \frac{\left\langle\kappa_{s}^{2}\right\rangle_{\mathrm{alk}}-\left\langle\kappa_{s}^{2}\right\rangle_{w}}{R T} .
$$

If the contributions of fluctuations in water and alkane cancel, or if they are insignificant, then the enthalpy of transfer has the usual meaning of the difference of average potential energies

$$
\Delta H_{0} \cong\left\langle E_{s, \text { alk }}^{\text {attract }}\right\rangle-\left\langle E_{s, w}^{\text {attract }}\right\rangle .
$$

Enthalpy changes for benzene, 3MI and TriBP are listed in Tables I and II. The curvature in temperature dependence of energy of ESE for TeCP requires introduction of additional process.

It follows from comparison of entropy terms in Eq. (31) and Eq. (30) that the entropy contribution to environmental swap energy, $-T \Delta S_{0}$, is equal to $T \Delta S_{\mathrm{cfg}, w}$ meaning that the measured $\Delta S_{0}=-\Delta S_{\mathrm{cfg}, w}$. Additional insight can be derived from Huang and Chandler's work ${ }^{28}$ containing computed temperature dependence of solvation of free energy in water for a spherical solute of radius $0.33 \mathrm{~nm}$. These data can be used as a reference for molecules in the present study. The computed results confirmed the expectation that attractive interactions influence primarily enthalpy, but not the entropy of solvation, an effect predicted earlier. ${ }^{28}$ It follows from the temperature dependence of solvation free energy of a 0.33 nm sphere [Fig. 7 in Huang and Chandler (Ref. 28)] that the cavity entropy term used in the above model $\Delta S_{\mathrm{cfg}, w}$ $=-46 \mathrm{~J} \mathrm{~mol}^{-1} \mathrm{~K}^{-1}$ which is close to the value of entropy change, $\Delta S_{0}$, obtained from the temperature dependence of environmental swap energy of benzene, TeCP and TriBP. The measured values of $\Delta S_{0}$ are $+48.9 \pm 1.4 \mathrm{~J} \mathrm{~mol}^{-1} \mathrm{~K}^{-1}$ for benzene, $+47.1 \pm 4.2$ for $\mathrm{TeCP}$, and $+41.6 \pm 5.4$ for TriBP. Thus the entropy of transfer of benzene, TeCP and TriBP into alkane is dominated by the collapse of the cavity in water. 
In the above cavity model we assumed that the entropy of cavity formation in alkane is due to configurational entropy of alkane chains,

$$
\Delta S_{\mathrm{cfg}, \mathrm{alk}}=-R\left(1-\frac{V_{s}^{0}}{V_{\mathrm{alk}}^{0}}\right) .
$$

Equation (34) predicted correctly the dependence of free energy of transfer of benzene on the number of carbons in alkane chains as shown in Fig. 2(a). Experimental results for $3 \mathrm{MI}, \mathrm{TeCP}$, and TriBP are consistent with $\Delta S_{\mathrm{cfg}, \text { alk }}=0$. The physical origin of the absence of configurational entropy effect of alkane chains for $3 \mathrm{MI}, \mathrm{TeCP}$, and TriBP is not clear, it may be associated with their size and polarity.

In terms of FH model, the absence of the configurational entropy component for $3 \mathrm{MI}, \mathrm{TeCP}$, and TriBP in alkanes suggests that the total interaction between these solutes and alkanes does not cause significant restriction of conformations of alkanes surrounding these solutes. One possibility is that the distribution of our solutes and alkane chains is not random, which is one of the assumptions of FH model. Pictorially, in contrast to benzene, alkanes do not "wrap" around 3MI, TeCP, and TriBP and thus do not reduce their configuration space. One possible reason is the presence of water associated with these molecules as they transfer into alkane. Energy of hydrogen bonds is known to be several times greater than the thermal energy and so it is conceivable that hydrogen-bonded water associated with $-\mathrm{NH}$ or $-\mathrm{OH}$ groups of 3MI, TeCP, or TriBP would co-transfer with these solutes into the alkane phase. The co-transfer of solvated water into cyclohexane has been documented for $3 \mathrm{MI} .{ }^{8}$ The consequence of water-solvated $3 \mathrm{MI}, \mathrm{TeCP}$, and TriBP may result in the destruction of randomness of alkane phase assumed in Flory's model. Diminished "wrapping" diminishes the configurational entropy effect.

Another possibility is that local fluctuations of attractive interactions between the solute and alkane, associated with thermal motion and the uneven charge distribution modulate the alkane-solute separation. This also may effectively remove the restrictions on the configuration space of alkane chains. There is a need to explore further whether these fluctuations can bring about entropy-enthalpy compensation in which $T \Delta S_{\text {cfg }}$ is removed from the energy of solvation by $\Delta H_{\text {cfg }}$ due to alkane reorganization.

The above effects are important for understanding the state of molecules in hydrophobic environments and at molecular interfaces that play critical role in interaction of drugs with receptors as well as in molecular imprinting.

Our finding that the contribution of configurational entropy on transfer of $3 \mathrm{MI}, \mathrm{TeCP}$, and TriBP into alkanes is unmeasurable is pertinent to current theoretical developments of understanding of solvation. One of the conceptual issues is the magnitude of the volume-dependent contribution to the solvation free energy. In the present work these effects were accounted for by Flory's model via Eq. (12) for benzene, but were absent for other solutes. Shimizu and co-workers ${ }^{29}$ analyzed solvation of fused hard spheres using a continuum model (rather than a lattice model), and concluded that solute-volume term is small even for flexible chain solvents, which is interesting. This property is consis- tent with our finding for 3MI, TeCP, and TriBP even though we seek an explanation in terms of the Flory's model. There are also novel conceptual and experimental issues of how to determine properly the environmental swap energy that would be free of volume contributions. Vitha and Carr ${ }^{30}$ analyzed transfer of an ideal point solute between two immiscible van der Waals solvents of different size and demonstrated that the free energy of transfer would be nonzero if calculated either from the mole fraction- or from the number density-based partition coefficient. Both ESE's would contain, in principle, entropic contributions due to the difference in free volumes in partition media. Properties of their model have demonstrated the importance of molecular sizes and therefore of molar volumes of partition media for the energetics of transfer of solutes. They also resulted in a proposal to adopt a new condition of equilibrium based on free volume concentrations. Thus in addition to mole fraction- and number density-based partition coefficients a new type of partition coefficient has been proposed. At this time the free volume partition coefficients are of primarily theoretical interest since it is not known how the free volume is distributed and what fraction of the free volume would be accessible to a particular solute. At present there is no method available to convert experimental values of conventional partition coefficients into free volume partition coefficients. Furthermore, there is also a possibility that environmental swap energy based on the difference of solvation Gibbs energies as defined by Eq. (11) is free of the volume artifacts. In view of these uncertainties it is most appropriate to continue to interpret experimental results in terms of number density, or molarity-based partition coefficients and use the concept of solvation Gibbs energy. Another generalization, supported by experimental results of this work, is that one cannot indiscriminately use Flory-Huggins models to account for configurational entropy effects because these effects may not be present in the particular system. For benzene the contribution of configurational entropy of alkanes could be isolated from the free energy of transfer whereas for $3 \mathrm{MI}, \mathrm{TeCP}$, and TriBP there is no experimental evidence for its presence.

\section{ACKNOWLEDGMENTS}

This work was partially supported by NIH Grant No. 1R15GM57634-01. We would like to thank Xavier Siebert for stimulating discussion of entropic effects and Mingdi Yan for introduction to molecular imprinting.

${ }^{1}$ C. Tanford, J. Phys. Chem. 83, 1802 (1979).

${ }^{2}$ K. A. Sharp, A. Nicholls, R. Friedman, and B. Honig, Biochemistry 30, 9686 (1991)

${ }^{3}$ I. Tunon, E. Silla, and J. L. Pascual-Ahuir, Protein Eng. 5, 715 (1992).

${ }^{4}$ H. S. Ashbaugh, E. W. Kaler, and M. E. Paulaitis, J. Am. Chem. Soc. 121, 9243 (1999)

${ }^{5}$ K. A. Sharp, A. Nicholls, R. F. Fine, and B. Honig, Science 252, 106 (1991).

${ }^{6}$ E. Gallicchio, M. M. Kubo, and R. M. Levy, J. Phys. Chem. B 104, 6271 (2000).

${ }^{7}$ K. S. Rogers and A. Cammarata, J. Med. Chem. 12, 692 (1969).

${ }^{8}$ W. C. Wimley and S. H. White, Biochemistry 31, 12813 (1992).

${ }^{9}$ L. S. Yaguzhinsky, E. G. Smirnova, L. A. Ratnikova, G. M. Kolesova, and I. P. Krasinskaya, Bioenergetics 5, 163 (1973).

${ }^{10}$ J. Saarikoski and M. Viluksela, Ecotoxicol. Environ. Saf. 6, 501 (1982). 
${ }^{11}$ B. I. Escher and R. P. Schwarzenbach, Environ. Sci. Technol. 30, 260 (1996).

${ }^{12}$ B. I. Escher, M. Snozzi, and R. P. Schwarzenbach, Environ. Sci. Technol. 30, 3071 (1996).

${ }^{13}$ B. I. Escher, R. Hunziker, R. P. Schwarzenbach, and J. C. Westall, Environ. Sci. Technol. 33, 560 (1999).

${ }^{14}$ G. L. Pollack and J. F. Himm, J. Chem. Phys. 77, 3221 (1982).

${ }^{15}$ A. Ben-Naim and Y. Marcus, J. Chem. Phys. 80, 4438 (1984).

${ }^{16}$ L. R. De Young and K. A. Dill, J. Phys. Chem. 94, 801 (1990).

${ }^{17}$ H. S. Chan and K. A. Dill, Annu. Rev. Biophys. Biomol. Struct. 26, 425 (1997).

${ }^{18}$ C. D. Eads, J. Phys. Chem. B 103, 5869 (1999).

${ }^{19}$ A. Ben-Naim, J. Phys. Chem. 82, 792 (1978).

${ }^{20}$ A. Ben-Naim, J. Phys. Chem. 83, 1803 (1979).
${ }^{21}$ A. Holtzer, Biopolymers 32, 711 (1992).

${ }^{22}$ A. Holtzer, Biopolymers 34, 315 (1994).

${ }^{23}$ A. Ben-Naim, J. Solution Chem. 30, 475 (2001).

${ }^{24}$ M. L. Huggins, J. Chem. Phys. 9, 440 (1941).

${ }^{25}$ P. J. Flory, Principles of Polymer Chemistry (Cornell University Press, Ithaca, NY, 1953).

${ }^{26} \mathrm{C}$. Hansch and A. Leo, Exploring QSAR: Fundamentals and Applications in Chemistry and Biology (American Chemical Society, Washington, DC, 1995).

${ }^{27}$ G. Graziano, Can. J. Chem. 80, 401 (2002).

${ }^{28}$ D. M. Huang and D. Chandler, J. Phys. Chem. B 106, 2047 (2002).

${ }^{29}$ S. Shimizu, M. Ikeguchi, S. Nakamura, and K. Shimizu, J. Chem. Phys. 110, 2971 (1999).

${ }^{30}$ M. F. Vitha and P. W. Carr, J. Phys. Chem. B 104, 5343 (2000). 